\title{
Energetic Ion Measurements Using a Directional Probe
}

\author{
Kenichi NAGAOKA, Mitsutaka ISOBE, Kouji SHINOHARA ${ }^{1)}$, Masaki OSAKABE, \\ Akihiro SHIMIZU and Shoichi OKAMURA \\ National Institute for Fusion Science, Toki 509-5292, Japan \\ 1) Japan Atomic Energy Agency, Naka 311-0193, Japan
}

(Received 5 December 2005 / Accepted 21 December 2005)

\begin{abstract}
A directional Langmuir probe (DLP) method has been applied to measurement of energetic particles (ions) in a magnetically confined plasma. Two experimental demonstrations were performed in the compact helical system (CHS). One was a neutral beam modulation experiment and the other was the measurement of energetic ion loss induced by MHD bursts. The results of the DLP were consistent with those of a neutral particle analyzer (NPA) and a lost ion probe (LIP). These experiments show that this method is applicable outside and also inside the last closed flux surface.

(c) 2006 The Japan Society of Plasma Science and Nuclear Fusion Research
\end{abstract}

Keywords: energetic particle transport, directional probe method, energetic particle mode

DOI: $10.1585 /$ pfr. 1.005

Energetic particle (ion) transport is one of the most important issues for burning fusion plasmas, such as those in the international thermonuclear experimental reactor (ITER). In particular, the interaction between energetic particles and MHD instabilities is a key issue for energetic particle confinement. Significant enhancement of energetic particle loss due to MHD instabilities has been experimentally observed in many fusion devices, and MHD instabilities have been intensively investigated both experimentally and theoretically [1]. One of the recent topics in these researches is the identification of an energeticparticle distribution function that is very sensitive to the excitation of MHD instabilities [2]. However, it is difficult to observe the spatial distribution of energetic particles in core plasmas by conventional diagnostic tools such as a neutral particle analyzer (NPA) because of insufficient spatial resolution. In this paper, the application of a directional Langmuir probe (DLP) method for energetic particle measurement is proposed in order to measure energetic particles with high spatial resolution, and two experimental demonstrations of this method are presented.

DLP is useful in obtaining deviations of the ion (electron) velocity distribution function from the dependence of the ion (electron) current on the angle of the normal vector of the particle collecting surface. The DLP method is well known as a plasma flow measurement method [3-6], and is also utilized to measure the anisotropic electron energy distribution function [6,7]. Moreover, the DLP method has sensitivity to beam components of ions and electrons in cases where the beam component is relatively stronger than the flow component: for example, toroidal plasmas sustained by a tangentially injected neutral beam. In the case of co-going strong suprathermal ion beams, the energetic

author's e-mail: nagaoka@lhd.nifs.ac.jp particle flux, $I_{\mathrm{b}}$, is approximately given by the difference between the co-going ion flux, $I_{\mathrm{co}}$, and the counter-going ion flux, $I_{\mathrm{ctr}}$, measured by a DLP:

$$
I_{\mathrm{b}} \sim I_{\mathrm{co}}-I_{\mathrm{ctr}} \text {. }
$$

The DLP used in this experiment consists of tenchannel probe array. The DLP has been vertically installed from the top in a horizontally elongated cross-section of $\mathrm{CHS}$, and it can move in the vertical, $z$, and major radius, $R$, directions. Moreover, this probe can be rotated along the probe axis, so it can measure co- and ctr-going fluxes separately. The probe is $40 \mathrm{~mm}$ in diameter, while the surface area of the electrodes is $4 \mathrm{~mm}$ in diameter. The spatial resolution of this probe is $4 \mathrm{~mm}$. The sample speed of this probe system is $1 \mathrm{MS} / \mathrm{sec}$, and the time resolution is $<10 \mu \mathrm{sec}$. In order to collect the ion current in energetic ion measurement, a voltage of $-120 \mathrm{~V}$ is applied to each electrode of the DLP.

The neutral beam modulation experiment was performed in CHS, and the modulation pattern and the probe currents are shown in Fig.1-(a) and -(b), respectively. The co-going flux measured by the DLP, $I_{\mathrm{co}}$, at the position of $r / a=0.84$ was larger than the ctr-going one, $I_{\mathrm{ctr}}$, in the NBI heating phase, while the two fluxes were the same in the electron cyclotron heating $(\mathrm{ECH})$ phase. This difference in the two fluxes implies high-energy particle flux, because the NBI is injected into the co-direction in CHS. The energetic particle flux given by eq. (1) (shown in Fig.1(c)) agrees well with the neutral particle flux measured by a neutral particle analyzer (Fig.1-(d)).

Excitations of toroidicity-induced Alfven eigenmodes (TAE) and energetic particle modes (EPM) have been observed [8], and energetic particle losses synchronized with EPM bursts have also been observed by a lost ion probe 


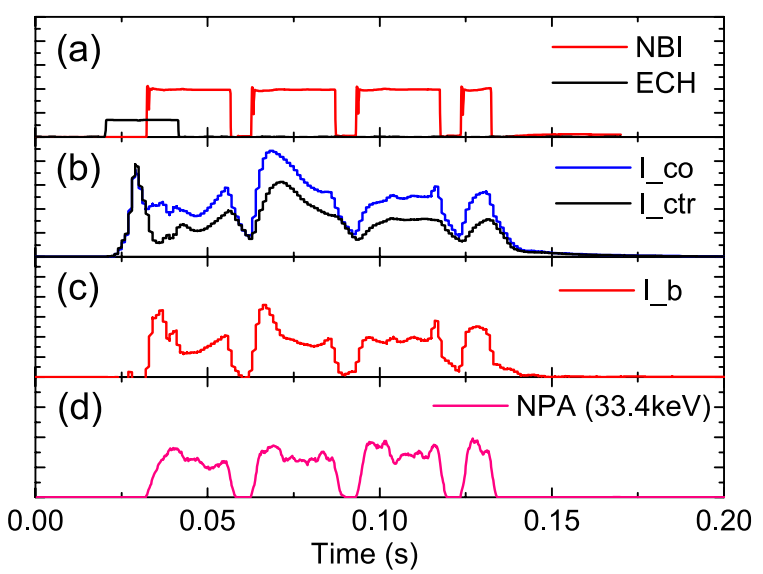

Fig. 1 The wave form of the neutral beam modulation experiment; (a) electron cyclotron heating and NBI heating, (b) ion currents measured by DLP, $I_{\mathrm{co}}$ and $I_{\mathrm{ctr}}$, (c), the difference between $I_{\mathrm{co}}$ and $I_{\mathrm{ctr}}$, and (d) neutral particle flux with the energy of $33.4 \mathrm{keV}$ measured by a neutral particle analyzer.

(LIP) in NBI plasmas in CHS $[9,10]$. The energetic particle measurements by the DLP were performed in plasmas with excited EPM bursts. Significant increases of the co-going flux measured by the DLP were observed in the NBI heating phase $(80 \mathrm{msec}<t<180 \mathrm{msec})$, while no response was observed in the ctr-going flux, as shown in Fig.2. This response of the co-going flux to the EPM bursts was observed in the region of $0.8<r / a<1.05$ (which is limited by the probe drive system). Outside the LCFS, the responses observed by the DLP agreed well with the LIP observation. These results indicate that the responses of the co-going flux measured by the DLP are those of energetic particles to EPM bursts; detailed analysis of this result will be shown elsewhere.

In conclusion, the local measurement of energetic ions using a DLP was experimentally demonstrated, and these results agreed well with NPA/LIP observations. The significant advantage of the DLP method is its high spatial resolution inside the LCFS. Observation of the distribution function of the energetic particles and investigations of the energetic particle transport induced by TAE and/or EPM are the next steps of this study.

The authors would like to thank Profs. Toi (NIFS) and

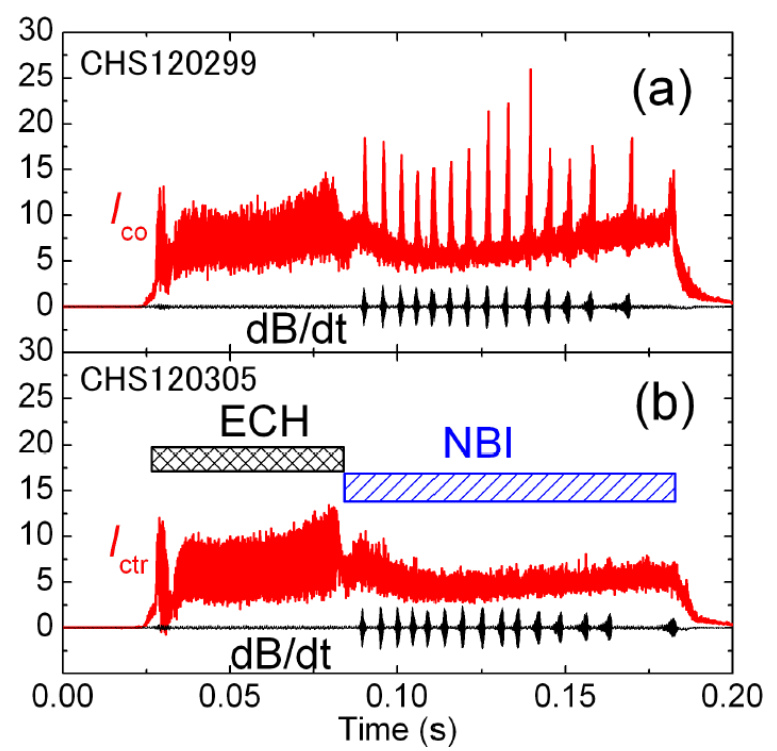

Fig. 2 (a) The co-going and (b) the ctr-going ion fluxes measured by the DLP. The signal of the magnetic coil is also shown.

Y. Todo (NIFS) for fruitful discussions. This work was supported by a Grant-in-Aid of the National Institute for Fusion Science (NIFS05ULPD609) and by a Grant-in-Aid of the Ministry of Education, Culture, Sports, Science and Technology of Japan (15740333).

[1] W.W. Heidbrink and G.J. Sadler, Nucl. Fusion 34, 535 (1994).

[2] W.W. Heidbrink, Phys. Plasmas 9, 2113 (2002).

[3] M. Hudis and L. M. Lidsky, J. Appl. Phys. 41, 5011 (1970).

[4] K. Nagaoka, A. Okamoto, S. Yoshimura and M.Y. Tanaka, J. Phys Soc. Jpn. 70, 131 (2001).

[5] A. Ando, T. Watanabe, H. Tobari, K. Hattori and M. Inutake, J. Plasma Fusion Res. 81, 451 (2005).

[6] T. Shikama, S. Kado, S. Kajita and S. Tanaka, J. J. Appl. Phys. 43, 809 (2004).

[7] S. Kado, T. Shikama, S. Kajita, T. Oishi and S. Tanaka, Contrib. Plasma Phys. 44, 656 (2004).

[8] K. Toi, S. Yamamoto, M. Takechi, N. Nakajima et al., J. Plasma Fusion Res. SERIES 5, 50 (2002).

[9] M. Isobe et al., Proceedings of 26th EPS Conf. on Contr. Fusion and Plasma Physics 23, 21 (1999).

[10] T. Kondo, M. Isobe et al., Nucl. Fusion 40, 1575 (2000). 